\title{
Considerations on Undistorted-Progressive $X$-Waves and Davydov Solitons, Fröhlich-Bose-Einstein Condensation, and Cherenkov-like effect in Biosystems
}

\author{
Marcus V. Mesquita, ${ }^{1}$ Áurea R. Vasconcellos, ${ }^{2}$ and Roberto Luzzi ${ }^{2}$ \\ 1. Instituto de Física, Universidade Federal do Ceará, 60455-760, Fortaleza, CE, Brazil \\ 2. Instituto de Física 'Gleb Wataghin', Universidade Estadual de Campinas, Unicamp, 13083-970 Campinas, SP, Brazil
}

Received on 3 June, 2003

\begin{abstract}
Research in ultrasonography has evidenced the propagation of a peculiar kind of excitation in fluids. Such excitation, dubbed a $\mathrm{X}$-wave, has characteristics resembling that of a solitary-wave type. We reconsider the problem in a medium consisting of a biological material of the like of $\alpha$-helix proteins. It can be shown that in this case is expected an excitation of the Davydov's solitary wave type, however strongly damped in normal conditions. The case of acetanilide, an organic polymer which resembles biopolymers, is considered, and the infrared spectrum analyzed. Davydov's soliton is evidenced as a coherent state of polar vibrations. The case of acoustic (sound) vibrations is also considered, where, also, a damped Davydov-like solitary wave may be excited. However, it is shown that when traveling in conditions sufficiently away from equilibrium, more precisely, when the soliton is embeded in the resulting Fröhlich-Bose-Einstein condensate, the lifetime of the solitary wave is largely enhanced. Moreover, a soliton moving in bulk with a velocity larger than that of the group velocity of the normal vibrational waves would produce a Cherenkov-like emission of phonons giving rise to the observed $\mathrm{X}$-wave-like pattern. This paper is a modified and extended version of an earlier publication on the subject.
\end{abstract}

\section{Introduction}

Experiments in ultrasonography have evidenced a particular kind of wave propagation, dubbed as $\mathrm{X}$-waves $[1,2]$. They have the characteristic of propagating without dispersion, and may result of relevance for ultrasound medical imaging. Later on, they were ascribed to some kind of the socalled undistorted propagating waves [3]. They are waves traveling in a material media, and the reported characteristics point to the possibility of they belonging to the category of solitary waves. Soliton is the name coined to describe a pulse-like nonlinear wave (the solitary wave referred to above) which emerges from a collision with a similar pulse having unchanged shape or speed. Its relevance in applied sciences has been described in a 1973 review paper by A. C. Scott et al. [4] Because of the above mentioned technologi$\mathrm{cal} /$ medical relevance we reanalyze the question in the case of propagation in biological materials.

The original category of solitary waves consists in the one observed by the Scottish engineer Scott-Russell in August 1834 in an English water channel, and reported in a 1844 meeting of the British Society for the Advancement of Science $[5,6]$. During the second half of this century many other types of solitary waves have been associated to a number of physical situations in condensed matter physics. Several, seemingly, have a fundamental role in important technological areas of large relevance for contemporary society. Among them we may highlight the case of doped organic polymers with very large conductivity for, e. g., use in very light, almost two-dimensional (sheets) batteries [7, 8, 9], and the case of propagation of light in optical fibers [10]. Another example is that of the so-called Davydov's solitons $[11,12]$, which may have a quite relevant role in bioenergetics.

Davydov's theory has received plenty of attention, and a long list of results published up to the first half of 1992 are discussed in a comprehensive review due to A. C. Scott [13]. As pointed out in that review, one question concerning Davydov's soliton is that of its stability at normal physiological conditions, that is, the ability of the excitation to transport energy (and so information) at long distances in the living organism, in spite of the relaxation mechanisms that are expected to damp it out at very short (micrometers) distances. On the assumption that the X-waves in ultrasonography may belong to the category of solitary waves in the material media, we consider this question on the basis of a model in nonequilibrium thermodynamic conditions, since any biological system is an open system out of thermal equilibrium. For that purpose we resort to an informational statistical thermodynamics (see for example [14-19]), based on the Nonequilibrium Statistical Operator Method (NESOM) [20-23]. The NESOM, which provides microscopic foundations to phenomenological irreversible thermodynamics [19], also allows for the construction of a nonlinear generalized quantum transport theory - a far-reaching generalization of the Chapman-Enskog's and Mori's methods - 
which describes the evolution of the system at the macroscopic level in arbitrary nonequilibrium situations [20,2325], a formalism to be used in what follows. We notice that the method has been applied with particular success to the study of optical and transport properties in solids [26] and also to modelled biopolymers [27, 28], of the kind we consider here, and to techno-industrial processes [29]. Consequently, we do not go over the details of the formalism, just adapting those earlier results for the present discussion, giving in each case the reference where they are reported. We separate the presentation into two parts: After a general derivation of the equations of evolution, a first analysis is concentrated on the case of polar vibrations (with frequencies in the infrared region of the spectrum), for which are available experimental results, particularly the case of acetanilide, which we analyze, comparing theory and experiment. This is done in subsection 2.1, while in subsection 2.2 we deal with the case of longitudinal acoustic vibrations.

As stated, we first consider a system where modes of polar vibrations are excited by a continuous supply of energy. These polar modes are coupled through a nonlinear kinetics with a bath consisting of a continuous medium modelled by a system of acoustic-like vibrations. The equations of evolution for the population of the vibrational modes are derived resorting to the nonlinear quantum kinetic theory that the NESOM provides. This corresponds to the description of polar vibrations of the CO-stretching type (Amide-I) in, for example, $\alpha$-helix proteins [11-13]. Experiments, of the class of Raman or neutron scattering or radiation absorption, in active biological systems are particularly difficult. For that reason, the comparison of the theory of next section with experiment is done in the case of the organic polymer acetanilide, which constitutes a good mimic of biopolymers [30]. The infrared spectrum in the frequency region corresponding to the CO-stretching oscillation is derived, and compare very well with the experimental measurements, confirming the presence of Davydov's soliton. Finally, we comment on the possibly very large increase of the lifetime of Davydov's soliton when propagating in an open medium sufficiently far from equilibrium.

In subsection 2.2, we consider a system of longitudinal acoustic vibrations in interaction with the above said thermal bath. We find a behavior of the acoustic modes quite similar to the one evidenced for the optical modes, namely, existence of the solitary-wave excitation, damped near equilibrium conditions but whose lifetime is greatly enhanced when propagating in a highly excited background.

Finally, it is demonstrated the possible emergence of a particular phenomenon, which we call Fröhlich-Cherenkov effect, consisting in that when the soliton is propagating with a velocity larger than the group velocity of the normal modes of vibration in the medium, a large number of phonons are emitted at a certain angle with the direction of propagation of the soliton.

\section{The Solitary Wave}

Let us consider a model biosystem which can sustain longitudinal vibrations and in interactions with a thermal bath of acoustic-like vibrations, which is described by the so-called Fröhlich-Davydov Hamiltonian given in [11] and [27]: This Hamiltonian is given in the so-called Random Wave Approximation, while the full Hamiltonian — to be used in what follows - is given by

$$
H=H_{0}+H_{I}=H_{0 S}+H_{0 B}+H_{I},
$$

where

$$
\begin{aligned}
& H_{0 S}=\sum_{\vec{q}} \hbar \omega_{\vec{q}}\left(a_{\vec{q}}^{\dagger} a_{\vec{q}}+\frac{1}{2}\right) \\
& H_{0 B}=\sum_{\vec{q}} \hbar \Omega_{\vec{q}}\left(b_{\vec{q}}^{\dagger} b_{\vec{q}}+\frac{1}{2}\right) \\
& H_{I}=\sum_{\vec{q}} Z_{\vec{q}} \varphi_{\vec{q}} a_{\vec{q}}^{\dagger}+\sum_{\vec{q}_{1} \vec{q}_{2}} V_{\vec{q}_{1} \vec{q}_{2}}^{(1)} a_{\vec{q}_{1}} b_{\vec{q}_{2}} b_{\vec{q}_{1}+\vec{q}_{2}}^{\dagger} \\
& +\sum_{\vec{q}_{1} \vec{q}_{2}} V_{\vec{q}_{1} \vec{q}_{2}}^{(1)} a_{\vec{q}_{1}} b_{\vec{q}_{2}}^{\dagger} b_{-\vec{q}_{1}+\vec{q}_{2}}+\sum_{\overrightarrow{q_{1} \vec{q}_{2}}} V_{\vec{q}_{1} \vec{q}_{2}}^{(1)} a_{\vec{q}_{1}} b_{\vec{q}_{2}} b_{-\vec{q}_{1}-\vec{q}_{2}} \\
& +\sum_{\vec{q}_{1} \vec{q}_{2}} V_{\vec{q}_{1} \vec{q}_{2}}^{(1)} a_{\vec{q}_{1}} b_{\vec{q}_{2}}^{\dagger} b_{\vec{q}_{1}-\vec{q}_{2}}^{\dagger}+\sum_{\vec{q}_{1} \vec{q}_{2}} V_{\vec{q}_{1} \vec{q}_{2}}^{(2)} a_{\vec{q}_{1}} a_{\vec{q}_{2}} b_{\vec{q}_{1}+\vec{q}_{2}}^{\dagger} \\
& +\sum_{\overrightarrow{q_{1}} \vec{q}_{2}} V_{\vec{q}_{1} \vec{q}_{2}}^{(2)} a_{\vec{q}_{1}} a_{\vec{q}_{2}} b_{-\vec{q}_{1}-\vec{q}_{2}}+\sum_{\overrightarrow{q_{1}} \vec{q}_{2}} V_{\vec{q}_{1} \vec{q}_{2}}^{(2)} a_{\vec{q}_{1}}^{\dagger} a_{\vec{q}_{2}} b_{\vec{q}_{1}-\vec{q}_{2}} \\
& +\sum_{\vec{q}_{1} \vec{q}_{2}} V_{\vec{q}_{1} \vec{q}_{2}}^{(2)} a_{\vec{q}_{1}} a_{\vec{q}_{2}}^{\dagger} b_{\vec{q}_{1}-\vec{q}_{2}}^{\dagger}+\text { H.c. }
\end{aligned}
$$

Figure 1 in [27] describes a particular biological system and the mechanical analog we are using. The Hamiltonian consists of the energy of the free subsystems, namely, that of the free vibrations, with $\omega_{\vec{q}}$ being their frequency dispersion relation ( $\vec{q}$ is a wave-vector running over the reciprocalspace Brillouin zone), and that of the thermal bath composed by oscillations with frequency dispersion relation $\Omega_{\vec{q}}$, with a Debye cut-off frequency $\Omega_{D}$. The interaction Hamiltonian $H_{I}$ contains the interaction of the system of polar vibrations with an external source [which pumps energy on the system and is the first term on the right side of Eq. (2c)], and, finally, the anharmonic interactions between both subsystems. The latter are composed of several contribution, namely, those associated with three quasi-particle (phonons) collisions involving one of the system and two of the thermal bath (we call $V_{\vec{q} q^{\prime}}^{(1)}$ the corresponding matrix element), and two of the system and one of the bath (we call $V_{\vec{q} \vec{q}^{\prime}}^{(2)}$ the corresponding matrix element). Finally, $a_{\vec{q}}\left(a_{\vec{q}}^{\dagger}\right), b_{\vec{q}}\left(b_{\vec{q}}^{\dagger}\right)$, are, as usual, annihilation (creation) operators of, respectively, normal-mode vibrations in the system and bath in mode $\vec{q}$, and the one corresponding to the quantum excitations in the pumping source, with $Z$ being the coupling strength. 


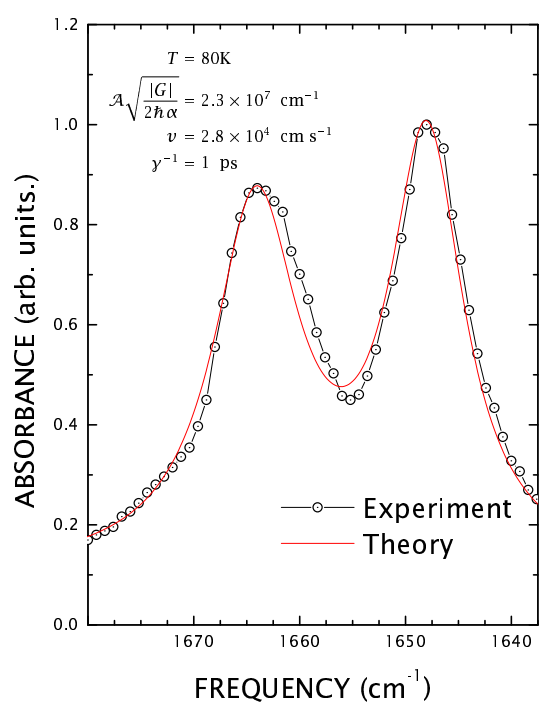

Figure 1. Optical vibrations: The IR absorption spectrum in acetanilide at $80 \mathrm{~K}$. Points are from the experimental data reported in [38], and the full line the calculation after Eq. (23).

Next step consists in the choice, within the tenets of NESOM, of the basic set of dynamical variables relevant for the present problem. Since we are dealing with excitation of vibrations in modes $\vec{q}$ (with energy $\hbar \omega_{\vec{q}}$ ), we need to introduce the number of excitations in each mode, $\hat{\nu}_{\vec{q}}=a_{\vec{q}}^{\dagger} a_{\vec{q}}$. Moreover, once the formation of a coherent state is expected (Davydov's soliton), we must introduce the field amplitudes $a_{\vec{q}}$ and $a_{\vec{q}}^{\dagger}$. Finally, since the thermal bath is taken as remaining constantly in a stationary state at a temperature $T_{0}$, via an efficient homeostatic mechanism, we introduce its Hamiltonian, $H_{B}$. The average of these quantities over the NESOM nonequilibrium ensemble constitute the basic set of macrovariables, which we designate as

$$
\left\{\nu_{\vec{q}}(t),\left\langle a_{\vec{q}}^{\dagger} \mid t\right\rangle,\left\langle a_{\vec{q}} \mid t\right\rangle, E_{B}\right\}
$$

that is, they define Gibbs's space of nonequilibrium thermodynamic states. This is the thermodynamic state space in Informational Statistical Thermodynamics (IST for short). IST is the thermodynamic theory for irreversible processes based on the nonequilibrium ensemble formalism NESOM $[20,21,22]$. The basic thermodynamic macrovariables of Eq. (3) are then given by

$$
\begin{gathered}
\nu_{\vec{q}}(t)=\operatorname{Tr}\left\{\hat{\nu}_{\vec{q}} \varrho(t)\right\} ; \\
\left\langle a_{\vec{q}} \mid t\right\rangle=\operatorname{Tr}\left\{a_{\vec{q}} \varrho(t)\right\} ; \\
\left\langle a_{\vec{q}}^{\dagger} \mid t\right\rangle=\left\langle a_{\vec{q}} \mid t\right\rangle^{*}=\operatorname{Tr}\left\{a_{\vec{q}}^{\dagger} \varrho(t)\right\} ; \\
E_{B}=\operatorname{Tr}\left\{\hat{H}_{B} \varrho_{B}\right\}
\end{gathered}
$$

where $\varrho(t)$ is the nonequilibrium statistical operator of the system, and $\varrho_{B}$ the canonical distribution of the thermal bath at temperature $T_{0}$. The statistical operator $\varrho(t)$ is taken in Zubarev's approach $[20,21]$, in this case given by

$$
\varrho(t)=\mathrm{e}^{-\hat{\mathrm{S}}_{\varepsilon}(\mathrm{t})}
$$

where

$$
\hat{S}_{\varepsilon}(t)=\hat{S}(t, 0)-\int_{-\infty}^{t} d t^{\prime} \mathrm{e}^{\varepsilon\left(\mathrm{t}^{\prime}-\mathrm{t}\right)} \frac{\mathrm{d}}{\mathrm{dt}^{\prime}} \hat{\mathrm{S}}\left(\mathrm{t}^{\prime}, \mathrm{t}^{\prime}-\mathrm{t}\right),
$$

with

$$
\hat{S}(t, 0)=\phi(t)+\sum_{\vec{q}}\left[F_{\vec{q}}(t) \hat{\nu}_{\vec{q}}+f_{\vec{q}}(t) a_{\vec{q}}+f_{\vec{q}}^{*}(t) a_{\vec{q}}^{\dagger}\right]
$$

being the so-called informational-statistical-entropy operator [31],

$$
\hat{S}\left(t^{\prime}, t^{\prime}-t\right)=\exp \left\{-\frac{1}{\mathrm{i} \hbar}\left(t^{\prime}-t\right) H\right\} \hat{S}(t, 0) \exp \left\{\frac{1}{\mathrm{i} \hbar}\left(t^{\prime}-t\right) H\right\},
$$

and $\varepsilon$ is a positive infinitesimal that goes to zero after the calculation of averages has been performed [20, 21]. Moreover, $\phi(t)$ - playing the role of a nonequilibrium partition function - ensures the normalization of the statistical operator.

In Eq. (6b) are present the Lagrange multipliers that the method introduces, namely those we have called

$$
\left\{F_{\vec{q}}(t), f_{\vec{q}}(t), f_{\vec{q}}^{*}(t), \beta_{0}\right\}
$$

where $\beta_{0}=\left(k_{B} T_{0}\right)^{-1}$, since the thermal bath remains in a stationary state at fixed temperature $T_{0}$, and the total statistical operator is the direct product of $\varrho(t)$ and $\varrho_{B} ; k_{B}$ is Boltzmann universal constant.

In continuation we proceed to derive, in the corresponding NESOM nonlinear quantum kinetic theory [20-25] the equations of motion for the basic variables of Eq. (2). Since $\beta_{0}$ is assumed to be constant in time, and so is $E_{B}$, we 
are simply left to calculate the equations of evolution for the population of the vibrational modes, $\nu_{\vec{q}}(t)$, and of the amplitude $\left\langle a_{\vec{q}} \mid t\right\rangle$ and its complex conjugate. As noticed, these equations are derived resorting to the nonlinear quantum generalized transport theory that the NESOM provides. We introduce an approximated treatment, however appropriate for the present case since the anharmonic interactions are weak, consisting of the so-called second order approximation in relaxation theory, SOART for short [24]. It is usually referred to as the quasi-linear theory of relaxation [32], which is a Markovian approximation involving only the second order in the interaction strengths [24], in the present case involving contributions proportional to $\left|V_{\vec{q} \vec{q}^{\prime}}^{(1)}\right|^{2}$ and $\left|V_{\vec{q} \vec{q}^{\prime}}^{(2)}\right|^{2}$.

The calculation shows that, because of the symmetry properties of the system and the selected choice of basic variables, several contributions in NESOM-SOART vanish in this case: The surviving one corresponds to the Golden Rule of quantum mechanics averaged over the nonequilibrium en- semble [27]. In compact form, the one for the population is

$$
\frac{d}{d t} \nu_{\vec{q}}(t)=I_{\vec{q}}+\sum_{j=1}^{5} J_{\vec{q}_{(j)}}(t)+\zeta_{\vec{q}}(t)
$$

where the first term on the right hand side is the one associated to the pumping source, where $I_{\vec{q}}$ is then the rate of population increase that it generates, and the remaining five collisions operators $J_{\vec{q}_{(i)}}(t)$ are those arising out of the anharmonic interactions. The first two correspond to collisional events involving a single vibration and two of the bath, and gives rise to a pure dissipative term which takes the form

$$
J_{\vec{q}_{(1)}}(t)+J_{\vec{q}_{(2)}}(t)=-\frac{1}{\tau_{\vec{q}}}\left[\nu_{\vec{q}}(t)-\nu_{\vec{q}}^{(0)}\right]
$$

where $\nu_{\vec{q}}^{(0)}$ is the population in equilibrium at temperature $T_{0}$, and $\tau$ plays the role of a relaxation time given by

$$
\tau_{\vec{q}}^{-1}=\frac{4 \pi}{\hbar^{2}} \frac{1}{\nu_{\vec{q}}^{(0)}} \sum_{\vec{q}^{\prime}}\left|V_{\vec{q} \vec{q}^{\prime}}^{(1)}\right|^{2} \nu_{\vec{q}^{\prime}}^{B} \nu_{\vec{q}-\vec{q}^{\prime}}^{B}\left[\delta\left(\Omega_{\vec{q}^{\prime}}+\Omega_{\vec{q}-\vec{q}^{\prime}}-\omega_{\vec{q}}\right)+2 \mathrm{e}^{\beta \hbar \Omega_{\tilde{q}^{\prime}}} \delta\left(\Omega_{\tilde{\mathrm{q}}^{\prime}}-\Omega_{\tilde{\mathrm{q}}-\tilde{\mathrm{q}}^{\prime}}+\omega_{\tilde{\mathrm{q}}}\right)\right],
$$

where $\nu_{\vec{q}}^{B}$ is the population of the phonons in the bath, namely, the Planck distribution

$$
\nu_{\vec{q}}^{B}=\left[\exp \left(\beta_{0} \hbar \Omega_{\vec{q}}\right)-1\right]^{-1},
$$

and the delta functions account for conservation of energy in the scattering events. The other terms, $J_{\vec{q}_{(i)}}(t)$ with $i=3,4,5$, are

$$
\begin{gathered}
J_{\vec{q}_{(3)}}(t)=\frac{8 \pi}{\hbar^{2}} \sum_{\vec{q}^{\prime}}\left|V_{\vec{q} \vec{q}^{\prime}}^{(2)}\right|^{2}\left[\nu_{\vec{q}-\vec{q}^{\prime}}^{B}\left(\nu_{\vec{q}^{\prime}}-\nu_{\vec{q}}\right)-\nu_{\vec{q}}\left(1+\nu_{\vec{q}^{\prime}}\right)\right] \delta\left(\Omega_{\vec{q}-\vec{q}^{\prime}}+\omega_{\vec{q}^{\prime}}-\omega_{\vec{q}}\right), \\
J_{\vec{q}_{(4)}}(t)=\frac{8 \pi}{\hbar^{2}} \sum_{\vec{q}^{\prime}}\left|V_{\vec{q} \vec{q}^{\prime}}^{(2)}\right|^{2}\left[\nu_{\vec{q}-\vec{q}^{\prime}}^{B}\left(\nu_{\vec{q}^{\prime}}-\nu_{\vec{q}}\right)+\nu_{\vec{q}^{\prime}}\left(1+\nu_{\vec{q}}\right)\right] \delta\left(\Omega_{\vec{q}-\vec{q}^{\prime}}-\omega_{\vec{q}^{\prime}}+\omega_{\vec{q}}\right), \\
J_{\vec{q}_{(5)}}(t)=\frac{8 \pi}{\hbar^{2}} \sum_{\vec{q}^{\prime}}\left|V_{\vec{q} \vec{q}^{\prime}}^{(2)}\right|^{2}\left[\nu_{\vec{q}+\vec{q}^{\prime}}^{B}\left(1+\nu_{\vec{q}^{\prime}}\right)-\left(\nu_{\vec{q}^{\prime}}-\nu_{\vec{q}+\vec{q}^{\prime}}^{B}\right) \nu_{\vec{q}}\right] \delta\left(\Omega_{\vec{q}+\vec{q}^{\prime}}-\omega_{\vec{q}^{\prime}}-\omega_{\vec{q}}\right),
\end{gathered}
$$

and, finally, the term $\zeta_{\vec{q}}(t)$ is the one which couples the populations with the amplitudes, namely

$$
\begin{gathered}
\zeta_{\vec{q}}(t)=\frac{4 \pi}{\hbar^{2}} \sum_{\vec{q}^{\prime}}\left|V_{\vec{q} \vec{q}^{\prime}}^{(1)}\right|^{2}\left|\left\langle a_{q} \mid t\right\rangle\right|^{2}\left\{\left(1+\nu_{\vec{q}^{\prime}}^{B}+\nu_{\vec{q}-\vec{q}^{\prime}}^{B}\right) \delta\left(\Omega_{\vec{q}-\vec{q}^{\prime}}+\Omega_{\vec{q}^{\prime}}-\omega_{\vec{q}^{\prime}}\right)\right. \\
\left.+2\left(\nu_{\vec{q}^{\prime}}^{B}-\nu_{\vec{q}-\vec{q}^{\prime}}^{B}\right) \delta\left(\Omega_{\vec{q}-\vec{q}^{\prime}}-\Omega_{\vec{q}^{\prime}}-\omega_{\vec{q}^{\prime}}\right)\right\} \\
+\frac{8 \pi}{\hbar^{2}} \sum_{\vec{q}^{\prime}}\left|V_{\vec{q} \vec{q}^{\prime}}^{(2)}\right|^{2}\left\{\left|\left\langle a_{q} \mid t\right\rangle\right|^{2}\left(1+\nu_{\vec{q}^{\prime}}+\nu_{\vec{q}-\vec{q}^{\prime}}^{B}\right)-\left|\left\langle a_{q^{\prime}} \mid t\right\rangle\right|^{2}\left(\nu_{\vec{q}}-\nu_{\vec{q}-\vec{q}^{\prime}}^{B}\right)\right\} \delta\left(\Omega_{\vec{q}-\vec{q}^{\prime}}+\omega_{\vec{q}^{\prime}}-\omega_{\vec{q}}\right) \\
-\frac{8 \pi}{\hbar^{2}} \sum_{\vec{q}^{\prime}}\left|V_{\vec{q} \vec{q}^{\prime}}^{(2)}\right|^{2}\left\{\left|\left\langle a_{q} \mid t\right\rangle\right|^{2}\left(\nu_{\vec{q}^{\prime}}-\nu_{\vec{q}-\vec{q}^{\prime}}^{B}\right)-\left|\left\langle a_{q^{\prime}} \mid t\right\rangle\right|^{2}\left(1+\nu_{\vec{q}}+\nu_{\vec{q}-\vec{q}^{\prime}}^{B}\right)\right\} \delta\left(\Omega_{\vec{q}-\vec{q}^{\prime}}-\omega_{\vec{q}^{\prime}}+\omega_{\vec{q}}\right) \\
+\frac{8 \pi}{\hbar^{2}} \sum_{\vec{q}^{\prime}}\left|V_{\vec{q} \vec{q}^{\prime}}^{(2)}\right|^{2}\left\{\left|\left\langle a_{q} \mid t\right\rangle\right|^{2}\left(\nu_{\vec{q}^{\prime}}-\nu_{\vec{q}+\vec{q}^{\prime}}^{B}\right)-\left|\left\langle a_{q^{\prime}} \mid t\right\rangle\right|^{2}\left(\nu_{\vec{q}}-\nu_{\vec{q}+\vec{q}^{\prime}}\right)\right\} \delta\left(\Omega_{\vec{q}+\vec{q}^{\prime}}-\omega_{\vec{q}^{\prime}}-\omega_{\vec{q}}\right) .
\end{gathered}
$$


The collision operator $J_{\vec{q}_{(5)}}$ is also a relaxation term (containing contributions nonlinear in the mode populations); and the first two terms are those responsible for the so-called Fröhlich effect, as a result that they account for, through the nonlinear terms, of the transfer of energy to the polar modes lowest in frequency. In fact, they contain nonlinear contributions proportional to

$$
\sum_{\vec{q}^{\prime}}\left|V_{\vec{q} \vec{q}^{\prime}}^{(2)}\right|^{2} \nu_{\vec{q}}(t) \nu_{\vec{q}^{\prime}}(t)\left[\delta\left(\Omega_{\vec{q}-\vec{q}^{\prime}}-\omega_{\vec{q}^{\prime}}+\omega_{\vec{q}}\right)-\delta\left(\Omega_{\vec{q}-\vec{q}^{\prime}}+\omega_{\vec{q}^{\prime}}-\omega_{\vec{q}}\right)\right],
$$

and we may notice that for modes $\vec{q}^{\prime}$ such that $\omega_{\vec{q}^{\prime}}>\omega_{\vec{q}}$ the energy conservation as required by the first delta function is satisfied, while this is not possible for the second: hence this nonlinear contribution tends to increase the population in mode $\vec{q}$ at the expenses of the other modes higher in frequency. Reciprocally, for $\omega_{\vec{q}^{\prime}}<\omega_{\vec{q}}$, the mode $\vec{q}$ transfers energy to the modes lower in frequency. Moreover, in
Eq. (12d) the term $\zeta_{\vec{q}}(t)$ acts as a source coupling the populations of the vibrational modes with the amplitudes of the expected coherent excitation (Davydov's soliton as shown a posteriori).

On the other hand, the equations of evolution for the field amplitudes are

$$
\begin{gathered}
\frac{\partial}{\partial t}\left\langle a_{\vec{q}} \mid t\right\rangle=-\mathrm{i} \tilde{\omega}_{\tilde{\mathrm{q}}}\left\langle\mathrm{a}_{\tilde{\mathrm{q}}} \mid \mathrm{t}\right\rangle-\Gamma_{\tilde{\mathrm{q}}}\left\langle\mathrm{a}_{\tilde{\mathrm{q}}} \mid \mathrm{t}\right\rangle+\Gamma_{\tilde{\mathrm{q}}}\left\langle\mathrm{a}_{\tilde{\mathrm{q}}} \mid \mathrm{t}\right\rangle^{*}-\mathrm{i} \mathrm{W}_{\tilde{\mathrm{q}}}\left\langle\mathrm{a}_{\tilde{\mathrm{q}}} \mid \mathrm{t}\right\rangle^{*}+ \\
+\sum_{\vec{q}_{1} \vec{q}_{2}} R_{\vec{q}_{1} \vec{q}_{2}}\left\langle a_{\vec{q}_{1}} \mid t\right\rangle\left\langle a_{\vec{q}_{2}}^{\dagger} \mid t\right\rangle\left(\left\langle a_{\vec{q}-\vec{q}_{1}+\vec{q}_{2}} \mid t\right\rangle+\left\langle a_{-\vec{q}+\vec{q}_{1}-\vec{q}_{2}}^{\dagger} \mid t\right\rangle\right), \\
\frac{\partial}{\partial t}\left\langle a_{\vec{q}}^{\dagger} \mid t\right\rangle=\text { the c.c. of the r.h.s. of Eq. (14a), }
\end{gathered}
$$

where $\tilde{\omega}_{\vec{q}}=\omega_{\vec{q}}+W_{\vec{q}}$, with $W_{\vec{q}}$ being a term of renormalization of frequency which will not be of interest in the following analysis, and the lengthy expression for $R_{\vec{q}_{1} \vec{q}_{2}}$ is given elsewhere [28]; its detailed expression is unnecessary for the analysis here. Finally, $\Gamma_{\vec{q}}(t)$, which has a quite relevant role in what follows, is given by

$$
\begin{aligned}
\Gamma_{\vec{q}}(t)= & \frac{1}{2} \tau_{\vec{q}}^{-1}(t)+\frac{4 \pi}{\hbar^{2}} \sum_{\vec{q}^{\prime}}\left|V_{\vec{q} \vec{q}^{\prime}}^{(2)}\right|^{2}\left[1+\nu_{\vec{q}^{\prime}}+\nu_{\vec{q}-\vec{q}^{\prime}}^{B}\right] \delta\left(\Omega_{\vec{q}-\vec{q}^{\prime}}+\omega_{\vec{q}^{\prime}}-\omega_{\vec{q}}\right)+ \\
& -\frac{4 \pi}{\hbar^{2}} \sum_{\vec{q}^{\prime}}\left|V_{\vec{q} \vec{q}^{\prime}}^{(2)}\right|^{2}\left[\nu_{\vec{q}^{\prime}}-\nu_{\vec{q}-\vec{q}^{\prime}}^{B}\right] \delta\left(\Omega_{\vec{q}-\vec{q}^{\prime}}-\omega_{\vec{q}^{\prime}}+\omega_{\vec{q}}\right)+ \\
& \frac{4 \pi}{\hbar^{2}} \sum_{\vec{q}^{\prime}}\left|V_{\vec{q} \vec{q}^{\prime}}^{(2)}\right|^{2}\left[\nu_{\vec{q}^{\prime}}-\nu_{\vec{q}+\vec{q}^{\prime}}^{B}\right] \delta\left(\Omega_{\vec{q}+\vec{q}^{\prime}}-\omega_{\vec{q}^{\prime}}-\omega_{\vec{q}}\right)
\end{aligned}
$$

The coupled equations (14) contain linear and tri-linear terms. Ignoring the latter, the resulting linearized equation has as solutions the normal damped wave motion, proceeding with a renormalized frequency and lifetime $\Gamma_{\vec{q}}^{-1}$. The complete equations, i.e. including the nonlinear terms, are of the Davydov's soliton type, but with damping, or more precisely, are nonlinear damped Schrödinger-like equations $[4,33]$. We introduce a representation in direct space, defining the averaged (over the nonequilibrium ensemble) field operator

$$
\psi(x, t)=\sum_{\vec{q}}\left\langle a_{\vec{q}} \mid t\right\rangle \mathrm{e}^{\mathrm{iqx}} .
$$

for linear propagation along the, say, $x$ direction on bulk or along the one-dimensional polymer.

\subsection{Polar (Optical) Vibrational Modes}

At this point we introduce a first type of analysis, specifying the vibrational modes as being of the class of lon- 
gitudinal polar-modes (optical modes with frequencies in the infrared), as in Davydov's and Fröhlich's works [11, $12,34,35]$. Their frequency dispersion relation is approximated (to a good degree of accuracy) by the parabolic-law $\omega_{\vec{q}}=\omega_{0}-\alpha q^{2}$, where $\omega_{0}$ and $\alpha$ are constants, standing for the frequency at the zone centre (the maximum one) and the curvature at this centre, respectively. Next, using Eqs. (14), after neglecting the coupling terms with the conjugated amplitude (what can be shown to be the case when we introduce from the outset a truncated Hamiltonian in the so-called Rotating Wave Approximation [36]), it follows that the average field amplitude satisfies the equation

$$
\begin{gathered}
\mathrm{i} \hbar \frac{\partial}{\partial \mathrm{t}} \psi(\mathrm{x}, \mathrm{t})=\left(\hbar \omega_{0}+\hbar \alpha \frac{\partial^{2}}{\partial \mathrm{x}^{2}}\right) \psi(\mathrm{x}, \mathrm{t})-\mathrm{i} \hbar \int_{0}^{\mathrm{L}} \frac{\mathrm{dx}^{\prime}}{\mathrm{L}} \Gamma\left(\mathrm{x}-\mathrm{x}^{\prime}\right) \psi\left(\mathrm{x}^{\prime}, \mathrm{t}\right)+ \\
+\int_{0}^{L} \frac{d x^{\prime}}{L} \int_{0}^{L} \frac{d x^{\prime \prime}}{L} \mathcal{R}\left(x-x^{\prime}, x-x^{\prime \prime}\right) \psi\left(x^{\prime}, t\right) \psi\left(x^{\prime \prime}, t\right) \psi^{*}(x, t),
\end{gathered}
$$

where $\Gamma$ and $\mathcal{R}$ are the back-transforms to direct space of $\Gamma_{\vec{q}}$ in Eq. (15) and $R_{\vec{q}_{1} \vec{q}_{2}}$ in Eq. (14a), and $L$ is the length of the sample. Moreover, we have taken a time-independent population $\nu_{\vec{q}}$, that is, according to Eq. (8) it is either the equilibrium distribution at temperature $T_{0}$ when no external pumping source is present (i. e. $I_{\vec{q}}\left(\omega_{\vec{q}}\right)=0$ ), or when in the presence of a constant pumping source leading, after a short transient has elapsed, to a steady state and, moreover, the term $\zeta_{\vec{q}}$ is weakly dependent on time (a condition to be characterized a posteriori).
Equation (17) is a nonlinear Schrödinger-type equation with damping [33]. Introducing a local approximation, that is, neglecting space correlations, after using the expressions

$$
\begin{gathered}
\mathcal{R}\left(x-x^{\prime}, x-x^{\prime \prime}\right)=\hbar G \delta\left(x-x^{\prime}\right) \delta\left(x-x^{\prime \prime}\right), \\
\Gamma\left(x-x^{\prime}\right)=\gamma_{s} \delta\left(x-x^{\prime}\right),
\end{gathered}
$$

we obtain that Eq. (17) becomes

$$
\mathrm{i} \frac{\partial \psi(\mathrm{x}, \mathrm{t})}{\partial \mathrm{t}}-\left(\omega_{0}-\mathrm{i} \gamma_{\mathrm{s}}\right) \psi(\mathrm{x}, \mathrm{t})-\alpha \frac{\partial^{2}}{\partial \mathrm{x}^{2}} \psi(\mathrm{x}, \mathrm{t})-\mathrm{G}|\psi(\mathrm{x}, \mathrm{t})|^{2} \psi(\mathrm{x}, \mathrm{t})=0
$$

Equations (14) and (19) are of the form of the equations derived by Davydov in an alternative way, but, with the present thermodynamic treatment clearly showing the damping effects. In equilibrium conditions at temperature $300 \mathrm{~K}$, the damping constants have values corresponding to lifetimes in the order of a few picoseconds. For the case of a Gaussian signal impinged at the beginning of the polymer chain, which can be approximated to a good degree of accuracy by a hyperbolic secant shape, and next using the Inverse Scattering Method [37] we obtain the solution

$$
\psi(x, t)=\mathcal{A} \exp \left\{\mathrm{i}\left[\frac{v}{2 \alpha} \mathrm{x}-\left(\omega_{\mathrm{s}}-\mathrm{i} \gamma_{\mathrm{s}}\right) \mathrm{t}-\frac{\theta}{2}\right]\right\} \operatorname{sech}\left[\mathcal{A}\left(\frac{|\mathrm{G}|}{2 \alpha}\right)^{1 / 2}(\mathrm{x}-v \mathrm{t})\right]
$$

where $\gamma_{s}$ is, evidently, the reciprocal lifetime of the excitation (taking $\gamma_{s}=0$ and $\omega_{0}=0$, Eq. (20) is the expression for Davydov's soliton in its original version [11, 12]), and we used $G=|G| \mathrm{e}^{\mathrm{i} \theta}$. Moreover,

$$
\omega_{s}=\omega_{0}-\frac{v^{2}}{4 \alpha}+\frac{|G| \mathcal{A}^{2}}{2},
$$

is the reciprocal period of the solitary wave and $\mathcal{A}$ and $v$ are an amplitude and a velocity of propagation fixed by the initial condition of excitation imposed by the external source.
Hence, it is proved the possible presence of Davydov's solitons in polymers, like the $\alpha$-helix protein in biological matter, but, we stress, of a damped character. The mechanism for the formation of the soliton is in this case interpreted as follows [13]: Vibrational energy of the COstretching (Amide-I) oscillators that is localized on the quasi-periodic helix acts - through a phonon coupling effect - to distort the structure of the helix. The helical distortion reacts - again through phonon coupling — to trap 
the Amide-I oscillation and prevents its dispersion in a selftrapping.

Let us consider the experimental observation of this excitation. As already noticed, experimental observation is difficult in active biological materials. A way around this difficulty consists into the experimental study of polymers whose vibronic characteristics resemble those of biopolymers, and a quite favorable one is acetanilide. Experiments of infrared absorption in acetanilide showed an "anomalous" band in the IR spectrum, which was ascribed to a Davydov's soliton [38], and later on reproduced in other experiments, and also observed in Raman scattering experiments [39-43].

We analyze the experiment of Careri et. al [38], resorting to a response function theory consistently derived in the framework of NESOM [20, 44, 45]. Without going into details (see [46]), the absorbance in the region of the CO-stretching mode has the expression

$$
\alpha(\omega)=\alpha_{n}(\omega)+\alpha_{s}(\omega)
$$

which describes two bands: one centered around $\omega_{\vec{q}}$, the frequency of the normal mode, ( $\vec{q}$ being equal to the IR-photon wave-vector) and with intensity proportional to the population in equilibrium $\nu_{\vec{q}}$ at temperature $T_{0}$, since no external pump is present, $I_{\vec{q}}=0$ in Eq. (8); and the other is centered around $\omega_{s}$, the frequency of the soliton. The band widths are $\gamma_{n}=\tau_{0}^{-1}$ and $\gamma_{s}=\tau_{0}^{-1}+\mathcal{A}(|G| / 2 \alpha)^{1 / 2}$, where $\tau_{0}^{-1}$ is the one of Eq. (10), for $\vec{q}$ near the zone center. Let us consider the experiments of reference [38], and take, for example, the case of $T_{0}=80 \mathrm{~K}$; on the basis of the red shift of the band due to the soliton in relation to the normal CO-stretching band, that is, $\omega_{0}-\omega_{s} \approx 16 \mathrm{~cm}^{-1}$, and that $\gamma_{s}-\gamma_{n} \approx$ $3.6 \mathrm{~cm}^{-1}$, we find that $\mathcal{A}(|G| / 2 \alpha)^{1 / 2} \approx 2.3 \times 10^{6} \mathrm{~cm}^{-1}$ and $v \approx 3 \times 10^{4} \mathrm{~cm} \mathrm{~s}^{-1}$. The calculated spectra is shown as a full line in Fig. 1, while the dots are experimental points, evidencing a satisfactory agreement.

In Fig. 2 it is shown the propagation of the energy accompanying the soliton (proportional to $|\psi(x, t)|^{2}$ ) along a few picoseconds after the application of the initial Gaussianlike excitation. It is clearly evidenced the conservation of the shape characteristic of the soliton, but accompanied, as already described, with a decay in amplitude in the picosecond range. Hence, a pulse signal impinged on the system would be carried a few micrometers, since the velocity of propagation is $\approx 3 \times 10^{4} \mathrm{~cm} \mathrm{~s}^{-1}$.

However, the situation may be substantially modified if the excitation propagates in a nonequilibrium background, namely the one provided by the presence of a constant pumping source $\left[I_{\vec{q}} \neq 0\right.$ in Eq. (8)] leading the system to a steady state, with populations, say, $\bar{\nu}_{\vec{q}}$, constant in time but much larger than the population in equilibrium. At a sufficient distance from equilibrium, as proposed by Fröhlich near thirty years ago $[34,35]$, and later on verified by several authors (in the framework of IST in [27]), is expected to arise a particular complex behavior in the system, namely a phenomenon akin to a Bose-Einstein condensation, consisting in that [because of the presence of the terms of Eq. (9)], and as already noticed, the energy stored in the polar vibrational modes is preferentially channeled to the modes lowest in frequency. The latter greatly increase in population, while

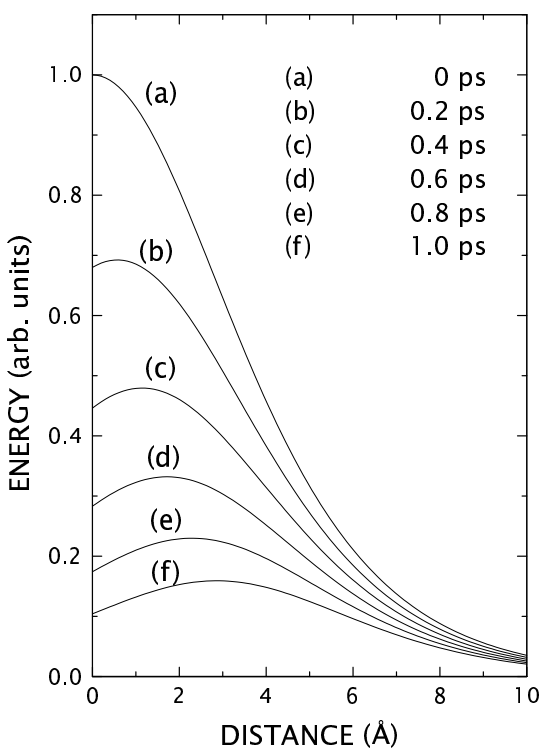

Figure 2. Optical vibrations: Profile of the soliton energy (proportional to $|\psi(x, t)|^{2}$ ) along a few picoseconds after application of the initial Gaussian-like perturbation (in the experimental conditions of Fig. 1 for $T=80 \mathrm{~K}$ ).

the modes at intermediate and high frequencies remain nearly constant on further increase of the intensity of the pump. As expressed above, this is similar to a Bose-Einstein condensation, but, it must be stressed, not in equilibrium but in nonequilibrium conditions, and then the phenomenon Fröhlich effect - may be considered a kind of emergence of a dissipative structure in Prigogine's sense [47, 48, 49]. This is illustrated in Fig. 3, where the steady state populations in terms of the intensity of the pumping source are shown. We have used numerical parameters characteristic of a polymer of the $\alpha$-helix protein type [27]. The figure evidences the large increase (after an intensity threshold has been attained) of a mode lowest in frequency (the one labelled 1), at the expenses of other modes higher in frequency. In Fig. 4 it is evidenced the Fröhlich-Bose-Einstein-like condensate at the lowest frequencies in the vibrational spectrum.

The relevant point to be stressed is that Fröhlich effect and Davydov soliton are phenomena arising out of the same nonlinear kinetic effects that are present in Eq. (9) for the populations $\nu_{\vec{q}}(t)$, and in Eq. (15) for the reciprocal lifetimes $\Gamma_{\vec{q}}$. As a consequence of the fact that, because of Fröhlich effect, the population of the modes lowest in frequency largely increase, concomitantly their lifetime also largely increases (i.e. the reciprocal lifetime $\Gamma_{\vec{q}}$ in Eq. (15) largely decreases), while for the modes at intermediate to high frequencies their lifetime largely decreases (the reciprocal of $\Gamma_{\vec{q}}$ largely increases). This is illustrated in Fig. 5 [28]. Hence, in the expression for the average field amplitude of Eq. (16), after a fraction of picosecond following the application of the exciting pulse has elapsed, there survive for a long time the contributions from the modes lowest in frequency, a survival time that keeps increasing as the 
intensity $I$ increases [28]. This implies that it may be expected that an excitation composed by a coherent interplay of the low-lying-in frequency excited polar (optical) modes in biopolymers, may propagate in the form of a Davydov solitary wave traveling undeformed and nearly undamped while Fröhlich condensate state is maintained.

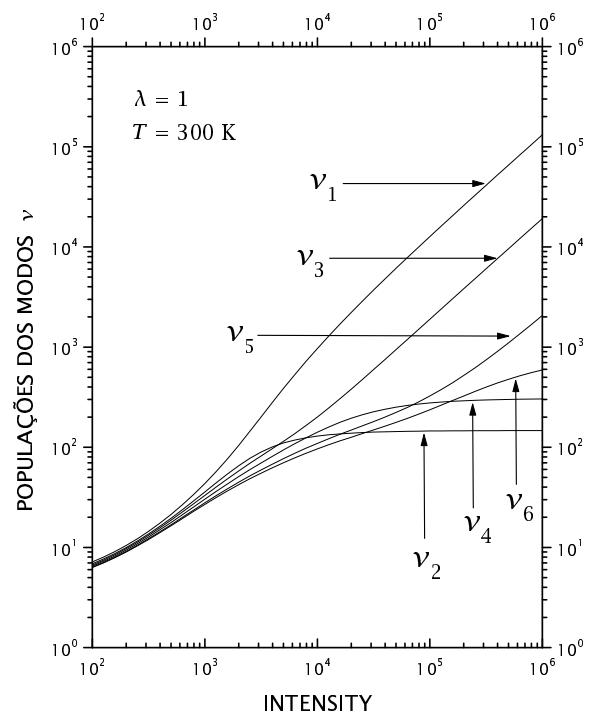

Figure 3. Optical vibrations: The steady-state populations vs. the intensity of the pumping source, for a selected set of modes: After an intensity $S=10^{3}$ is reached, there follows a large increase in the population $\nu_{6}$ of the mode lowest in frequency in the said set (After Ref. [27])

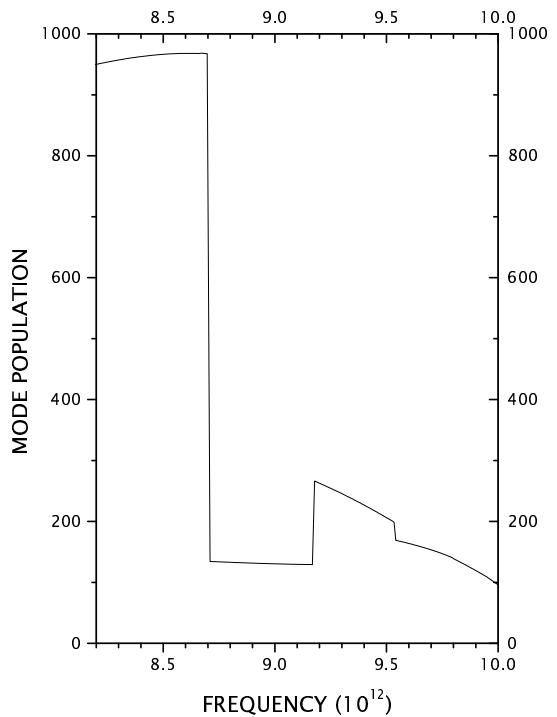

Figure 4. Optical vibrations: The steady-state populations for $S=10^{4}$, showing a "two fluid" separation consisting in the "condensate" at low frequencies and the "normal" contributions at higher frequencies (After Ref. [27]).

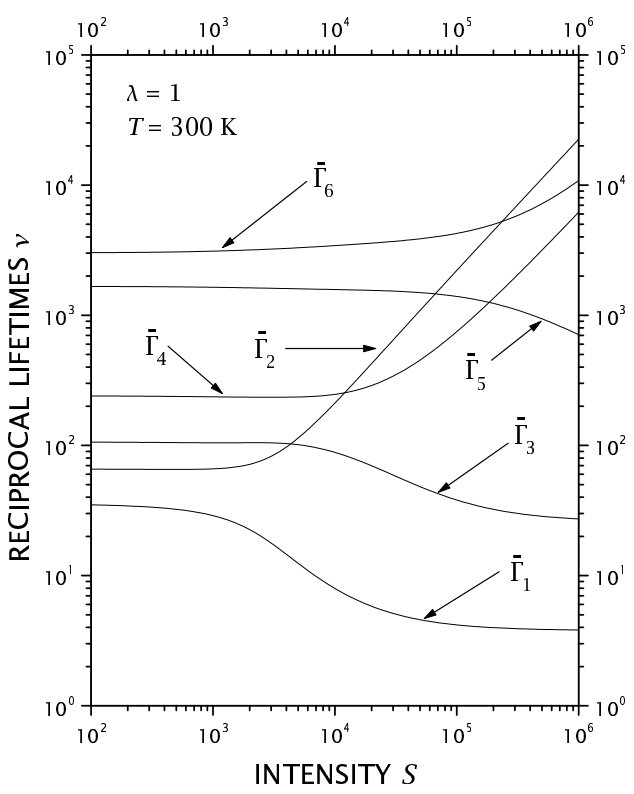

Figure 5. Optical vibrations: The lifetime of the same modes as in Fig. 3, in terms of the intensity of the pumping source.

\subsection{Acoustical Vibrational Modes}

So far we have considered propagation of vibronic waves in biological media, via Eq. (16), but restricted to the case of polar modes. We briefly consider next the case of longitudinal acoustic modes. For that purpose we return to Eqs. (14), where now we take into account that the dispersion relation $\omega_{\vec{q}}$ is, for acoustic-like vibrations, $s q$, where $s$ is the velocity of sound in the media, this meaning that we are using a Debye model. Using this dispersion relation, and the Ansatz that the excitation is expected to be a closed-packet solitary wave, we arrive at the equivalent of Eq. (19), in this case acquiring the expression

$$
\begin{gathered}
\mathrm{i} \frac{\partial}{\partial t} \psi(x, t)+\frac{\hbar}{2 M_{s}} \frac{\partial^{2}}{\partial x^{2}} \psi(x, t) \\
+\mathrm{i} \gamma_{s} \psi(x, t)-G_{s}|\psi(x, t)|^{2} \psi(x, t)=0
\end{gathered}
$$

as shown in Appendix A.

Evidently, this Eq. (23) is formally identical with Eq. (19) if in the latter we take $\omega_{0}=0$ and, of course with the coefficients being those corresponding to this case of LA (longitudinal acoustic) vibrations. However, a remarkable difference may be noticed, namely, while in Eq. (19) the coefficient in front of the second derivative in space is determined, through $\alpha$, by the bandwidth of the LO (longitudinal optical) vibrations dispersion relation, in this case, as shown in Appendix A, it depends through the pseudo-mass $M_{s}$ on the characteristics of the experiment, that is, depends on the width of the solitary wave packet which is determined by the initial condition. The solution for a given hyperbolic secantprofile signal impinged on the system, say, the same as in the previous subsection, is given by 


$$
\psi(x, t)=\mathcal{A} \exp \left\{\mathrm{i}\left[\frac{M_{s} v}{\hbar} x-\left(\omega_{s}-\mathrm{i} \gamma_{s}\right) t-\frac{\theta}{2}\right]\right\} \operatorname{sech}\left\{\mathcal{A}\left[\frac{\left|G_{s}\right| M_{s}}{\hbar}\right]^{1 / 2}(x-v t)\right\}
$$

where

$$
\omega_{s}=\frac{\left|G_{s}\right| \mathcal{A}^{2}}{2}-\frac{M_{s} v^{2}}{4 \hbar},
$$

As in the case of LO vibrations, the "acoustic" solitary wave is damped, and one may wonder if, as in the case of the "optical" solitary wave, this lifetime may be largely extended by the action of nonlinear kinetic terms enhanced by the pumping of energy on the system. We reconsider Eqs. (11), now specialized for the LA vibrations, and look for the stationary states when a constant exciting source is continuously applied.

To perform numerical calculations we choose a set of parameters in a typical order of magnitude approximation. We take for the Brillouin zone-end wavenumber $q_{B}=3.14 \times 10^{7} \mathrm{~cm}^{-1}$, $\omega_{\vec{q}}=s q \quad$ with $\quad s=1.8 \times 10^{5} \mathrm{~cm} \mathrm{~s}^{-1}, \quad \Omega_{\vec{q}}=s_{B} q$ with $s_{B}=1.4 \times 10^{5} \mathrm{~cm} \mathrm{~s}^{-1}$. Moreover, the matrix elements $V^{(1)}$ and $V^{(2)}$ are proportional to the square roots of the wavenumbers [50], say $V_{\vec{q} \vec{q}^{\prime}}^{(1,2)}=K^{(1,2)}\left[\left|\vec{q}_{1}\right|\left|\vec{q}_{2}\right|\left|\vec{q}_{1}-\vec{q}_{2}\right|\right]^{\frac{1}{2}}$, and $K^{(1)}$ is determined from a typical value of $10 \mathrm{ps}$ for the lifetime of Eq. (10) (for any system it can be determined from the linewidth in Raman scattering experiments). An open parameter $\lambda=\left|K^{(2)} / K^{(1)}\right|^{2}$ is introduced, and we take $\lambda=1$ to draw Fig. 6 . Finally, $L$, the length of the sample in the direction of propagation is taken as $10 \mathrm{~cm}$. Therefore, the permitted wavenumbers for propagation of vibrations are contained in the interval $\pi / L \leq q \leq q_{B}$. For these characteristic values it follows that, because of energy and momentum conservation in the scattering events, the set of equations of evolution, Eqs. (8), which in principle couple all modes among themselves, can be separated into independent sets each one having nine modes. For example, taking the mode with the lowest wavenumber $\pi / L$, the set to which it belongs contains the modes $\kappa^{n-1} \pi / L$, where $\kappa=\left(s+s_{B}\right) /\left(s-s_{B}\right)=8$ in this case, and $n=2,3, \ldots, 9$. Let us call $\nu_{1}, \ldots, \nu_{9}$ the corresponding populations, having frequencies $\omega_{1}=5.6 \times 10^{4} \mathrm{~Hz}$, $\omega_{2}=4.5 \times 10^{5} \mathrm{~Hz} . \omega_{3}=3.6 \times 10^{6} \mathrm{~Hz}, \omega_{4}=2.9 \times 10^{7} \mathrm{~Hz}$, $\omega_{5}=2.3 \times 10^{8} \mathrm{~Hz}, \omega_{6}=1.8 \times 10^{9} \mathrm{~Hz}, \omega_{7}=1.5 \times 10^{10} \mathrm{~Hz}$, $\omega_{8}=1.2 \times 10^{11} \mathrm{~Hz}, \omega_{9}=9.5 \times 10^{11} \mathrm{~Hz}$. Moreover, for illustration, the open parameter $\lambda$ is taken equal to 1 , and we consider that only the modes 2 and 3 (in the ultrasonic region) are pumped with the same constant intensity $S=I \bar{\tau}$, where $I_{2}=I_{3}=I$, and $I_{1}$ and $I_{n}$ with $n=4, \ldots, 9$ are null, and $\bar{\tau}$ is a characteristic time used for scaling purposes (as in [27]) here equal to $0.17 \mathrm{~s}$. The results are shown in Fig. 6, where it is evident the large enhancement of the population in the mode lowest in frequency $\left(\nu_{1}\right)$, for $S_{0} \simeq 10^{19}$, at the expenses of the two pumped modes $\nu_{2}$ and $\nu_{3}$, while the modes $\nu_{4}$ to $\nu_{9}$ (higher in frequency) are practically unaltered. The emergence of Fröhlich effect is clearly evidenced for this case of acoustical vibrations: In fact, pumping of the modes in a restricted ultrasonic band (in the present case in the interval $4.5 \times 10^{5} \mathrm{~Hz} \leq \omega \leq 2.8 \times 10^{7} \mathrm{~Hz}$ ), leads at sufficiently high intensity of excitation to the transmission of the pumped energy in these modes to those with lower frequencies $\left(\omega<\omega_{2}\right)$, while those with larger frequencies $\left(\omega>2.8 \times 10^{7} \mathrm{~Hz}\right)$ remain in near equilibrium, as shown in Fig. 7. It may be noticed that for the given value of $\bar{\tau}$, for $S=10^{23}$, the flux power provided by the external source, in the given interval of ultrasound frequencies being excited, is of the order of milliwatts. Modes in the interval $5.6 \times 10^{4} \leq \omega_{\vec{q}} \leq 4.5 \times 10^{5}$, those lowest in frequency, have large populations in comparison with those higher in frequency. As already noticed, because of these characteristics of Fröhlich's effect, it is sometimes referred-to as a Bose-Einstein-like condensation. However, it must be stressed that not in equilibrium, but in nonequilibrium conditions, then being a kind of nonequilibrium phase transition or better to say, a kind of emergence of a dissipative structure in Prigogine's sense [47-49]. Moreover, we may say, in a descriptive way, that it is present a kind of a "two fluid system", the normal one and the Fröhlich condensate.

Another relevant result is that, also as in the case of the optical vibrations, the modes in the condensate largely increase their lifetimes; this is shown in Fig. 8. Therefore, the soliton, composed by the coherent interplay of lowfrequency acoustical modes, travels nearly undamped in the Fröhlich condensate.

We proceed now to consider another possible novel phenomenon in this kind of systems.

\subsection{The accompanying Cherenkov-like emis- sion}

Considering either an "optical" or an "acoustical" soliton of the Davydov type respectively described in the previous subsections, we recall that the amplitude and the velocity of propagation are determined by the initial condition of excitation (that is, the energy and the momentum transferred in the process of interaction with the external source). For example, in the case of the acetanilide we have considered in subsection 2.1, and in the conditions of the experiment of Careri et al. [38], the velocity of propagation is larger than the group velocity of the phonons in the optical branch corresponding to the CO-stretching vibrations, which is small because the dispersion relation is flat. 


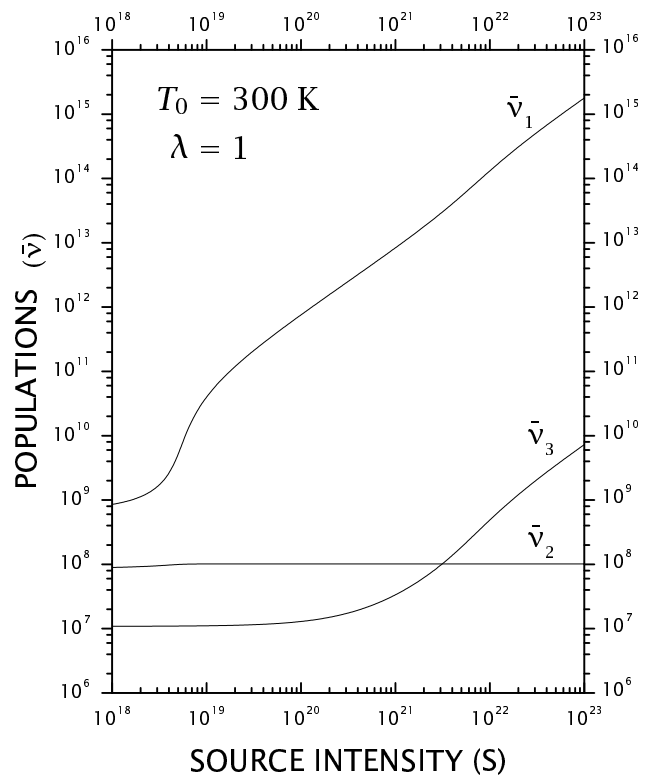

Figure 6. Acoustic vibrations: The steady state populations of the three relevant modes in the set - as described in the main text -, with increasing values of the intensity of the external source pumping modes labeled 2 and 3 in the ultrasonic region. (After Ref. [46]).

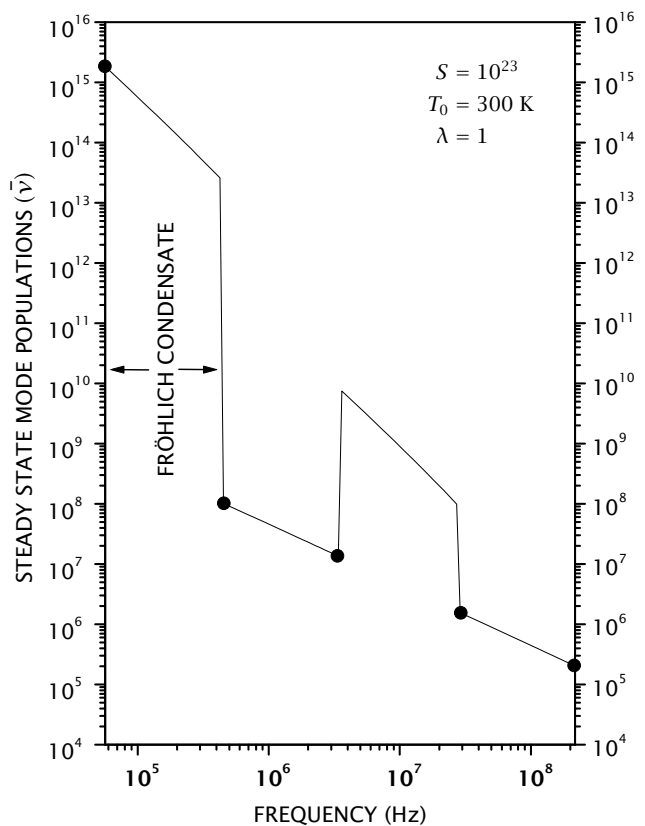

Figure 7. Acoustic vibrations: The population in the steady state for a pumping intensity $S=10^{23}$, of the modes along the spectrum of frequencies of the acoustic modes. Dots indicate the modes in the first set (the remaining part of the spectrum up to the highest Brillouin frequency $\omega_{B}=9.5 \times 10^{11} \mathrm{~Hz}$ has been omitted). (After Ref. [46]).

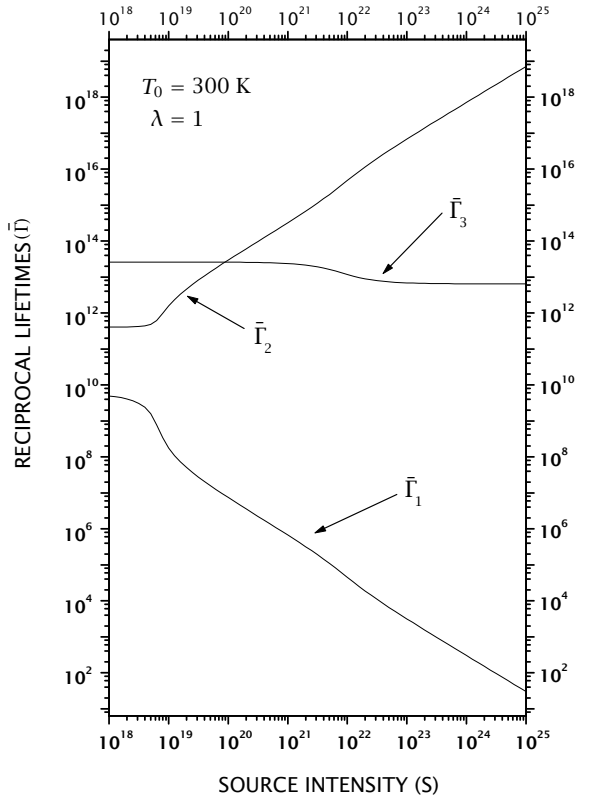

Figure 8. Acoustic vibrations: The lifetime of the same modes as in Fig. 7, in terms of the intensity of the pumping source (After Ref. [46]).

When the soliton velocity of propagation, say $v$, is larger than the group velocity of the normal vibrations, (the velocity of sound $s$ when the acoustic modes are involved), it may follow a Cherenkov-like effect. We recall that originally it was observed in electromagnetic radiation (e.g. [51]) by Cherenkov in 1934. It is a result that in a material media with an index of refraction $n$, the velocity of propagation of light is $c / n$, smaller than the velocity $c$ in vacuum (since $n>1$ ), and if an electron with velocity $u>c / n$ (but with the relativistic limitation of $u<c$ ) travels in this medium then, along a cone defined by the angle $\cos \theta=c / n u$ is emitted the so-called Cherenkov radiation: that is, along such direction photons are strongly emitted. This is the socalled superluminal radiation [52, 53].

Something similar is present in the case of phonons in the photoinjected plasma in semiconductors in the presence of an electric field: when the drift velocity of the carriers exceeds the group velocity of the $\vec{q}$-mode optical phonon, then along a cone whose axis is along the electric field, and with an aperture with angle $\theta_{\vec{q}}$ defined by

$$
\cos \theta=\omega_{\vec{q}} / v q,
$$

there follows a large emission of $\vec{q}$-mode optical phonons [54].

This is also the case when the soliton, either optical or acoustical, travels in bulk with a velocity $v$ larger than the group velocity of the normal vibronic waves. This is described elsewhere [46], and next we briefly outline the results. Inspection of Eq. (8) tells us that the presence of the direct coupling to the external source via $I_{\vec{q}}$, and indirectly through $\zeta$ depending on the squared amplitude of the soli- 
ton, tends to increase the population of phonons. But, as already noticed, because of Fröhlich effect, such pumped energy tends to concentrate in the modes lowest in frequency, those at the Brillouin zone boundary in the case of optical vibrations and around the zone center in the case of acoustic vibrations.

Take the case of acoustic phonons, when there should be a large increase in the population of the modes with very small wavenumber. A straightforward calculation of
Eq. (4a) leads to the result that

$$
\nu_{\vec{q}}(t)=\left[\mathrm{e}^{\mathrm{F}_{\tilde{\mathrm{q}}}}(\mathrm{t})-1\right]^{-1}+\left|\frac{\mathrm{f}_{\tilde{\mathrm{q}}}(\mathrm{t})}{\mathrm{F}_{\tilde{\mathrm{q}}}}\right|^{2} .
$$

Evidently, in the absence of the perturbation, that is, $I_{\vec{q}}=0$ and $\left\langle a_{\vec{q}}\right\rangle=0$ and then $f_{\vec{q}}=0$, it follows that $F_{\vec{q}}(t)=\hbar s q / k_{B} T_{0}$, and we recover the usual Planck distribution in equilibrium. In the presence of the perturbation we need to obtain both $F_{\vec{q}}(t)$ and $f_{\vec{q}}(t)$. On the one hand, a direct calculation tell us that

$$
\left|f_{\vec{q}}(t) / F_{\vec{q}}(t)\right|^{2}=\left|\left\langle a_{\vec{q}} \mid t\right\rangle\right|^{2} \approx \int_{0}^{L} \frac{d x}{L}|\psi(x, t)|^{2} \approx\left(\mathcal{A}^{2} w^{2} / L^{2}\right) \mathrm{e}^{-\gamma_{\mathrm{s}} \mathrm{t}}
$$

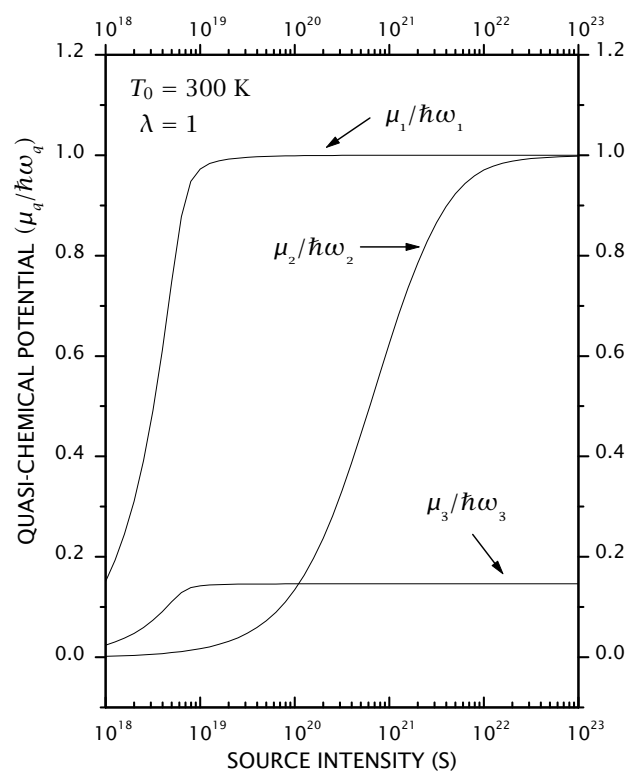

Figure 9. The quasi-chemical potential of the modes labeled 1 to 3 in Fig 6, with mode 1 corresponding to the one with the lowest frequency in the given set: it is evident the emergence of a "BoseEinstein-like condensation" for $S$ approaching a critical value of the order of $10^{19}$

where we have used Eqs. (16) and (20), and, we recall, under a sufficiently intense excitation $\gamma_{s}$ is small and then $\left|\left\langle a_{q} \mid t\right\rangle\right|^{2}$ becomes near time independent; we have called $w$ the width of the solitary wave packet.

On the other hand, $F_{\vec{q}}$ in steady state conditions after application of the constant external excitation, depends on the intensity of the pumping source. This Lagrange multiplier may be rewritten in either of two alternative forms, which resemble well known results in equilibrium theory. One is

$$
F_{\vec{q}}(t)=\left[\hbar s q-\mu_{\vec{q}}\right] / k_{B} T_{0},
$$

where $\mu_{\vec{q}}$ plays the role of a quasi-chemical potential (this kind of choice was done by Fröhlich [35] and Landsberg [55]). In Fig. 9 is shown the dependence of the quasichemical potential, corresponding to the modes in Fig. 6, with the pumping intensity. Another is

$$
F_{\vec{q}}=\hbar s q / k_{B} T_{\vec{q}}^{*}
$$

introducing a quasitemperature $T^{*}$, as it is done in semiconductor physics [26]. In Fig. 10 is shown the dependence of the dependence of the quasitemperature, corresponding to the modes in Fig. 6, with the pumping intensity.

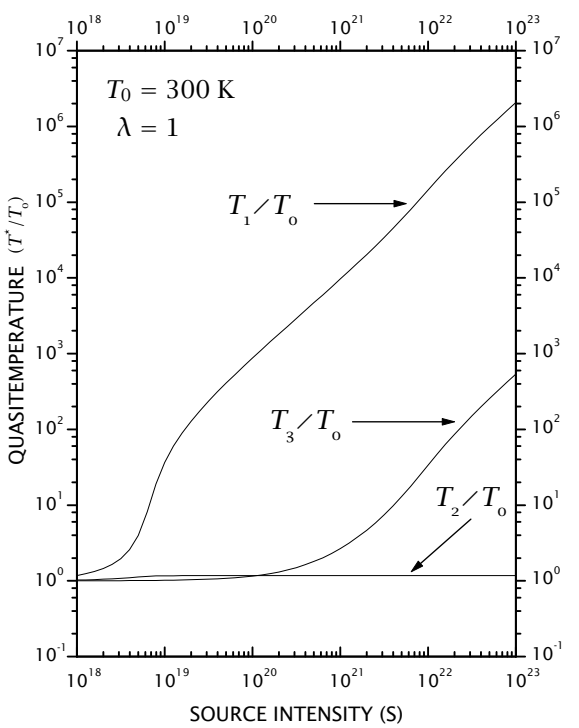

Figure 10. The quasi-temperature, defined in Eq. (30) for the modes in Fig. 6.

Let us take the choice of Eq. (30), then the quasitemperature $T^{*}$ is given by

$$
k_{B} T_{\vec{q}}^{*}=\hbar s q \ln \left[1+\frac{1}{\nu_{\vec{q}}-\left|\left\langle a_{\vec{q}}\right\rangle\right|^{2}}\right]
$$


and we recall that

$$
\left|\left\langle a_{\vec{q}}\right\rangle\right|^{2} \approx \mathcal{A}^{2} w^{2} / L^{2}
$$

(for $\gamma_{s} \rightarrow 0$, with $\nu_{\vec{q}}$ determined in each case solving
Eq. (8)). Using Eqs. (29) to (32) we have that [46]

$$
\mu_{\vec{q}}=\hbar v q \cos \eta_{\vec{q}}
$$

where

$$
\cos \eta_{\vec{q}}=\frac{s}{v}\left[1-\frac{k_{B} T_{0}}{\hbar s q} \ln \left[1+\left(\nu_{\vec{q}}-\left|\left\langle a_{\vec{q}}\right\rangle\right|^{2}\right)^{-1}\right]\right]=\frac{s}{v}\left[1-\frac{T_{0}}{T_{\vec{q}}^{*}}\right] .
$$

These results imply in this case in a phenomenon of a peculiar character which we call Frhlich-Cherenkov-effect. In fact, we note, first, that there follows a large enhancement of phonons in mode $\vec{q}$ for $\mu_{\vec{q}}$ approaching $\hbar s q$, and second, the linear motion of the soliton defines a particular direction, the one given by its velocity of propagation $\vec{v}$. Therefore, there is a preferential direction of production of vibrational waves given by

$$
\hbar s q \quad \approx \hbar v q \cos \eta_{\vec{q}}=\mu_{\vec{q}}
$$

or

$$
\cos \eta_{\vec{q}} \quad \approx s / v
$$

Equation (35) defines the direction of propagation $\vec{q}$ of the longitudinal vibration and its modulus. Since $\eta_{\vec{q}}$ depends only on the modulus of $\vec{q}$, there follows two Cherenkov-like privileged directions of emission of $\vec{q}$-mode phonons, one forward and one backwards, like the normal and anomalous Cherenkov cones in radiation theory as illustrated in Fig. 9 adapted from [53]. In the present case both directions are symmetrical on both sides of the centre defined at each time by the position occupied by the soliton. This may account for the observed so-called $\mathrm{X}$-waves $[1,56,3]$. In Fig. 12 is illustrated the cases of propagation of the normal sound wave (upper figure) and of the, presumably, solitary wavepacket selectively excited by the transducer (lower figure) with velocity larger than the sound velocity in the medium. The figure has appeared in [3]. Given the angle $\eta_{\vec{q}}$ (called the axicon angle in [1]), then $v$ is larger than $s$ in the percentage $\left[\left(v / \cos \eta_{\vec{q}}\right)-s\right] / v$. Same arguments are valid for the case of the optical soliton, when $\nabla_{\vec{q}} \omega_{\vec{q}}$ (the group velocity of the normal mode) enters in place of $s$. In the case of Fig. 12, a rough estimate gives $\eta \sim 13^{\circ}$ and $v_{s} / s \sim 1.02$, that is, the velocity of propagation of the soliton, $v$, is roughly $2 \%$ larger than the velocity of sound in the medium.

\section{Concluding remarks}

We have considered the propagation of vibronic excitations in nonlinear condensed matter media, like biological material. Because of the nonlinearities in the kinetic equations that describe the evolution of the macroscopic collective modes (nonlinearities having their origin in the microscopic

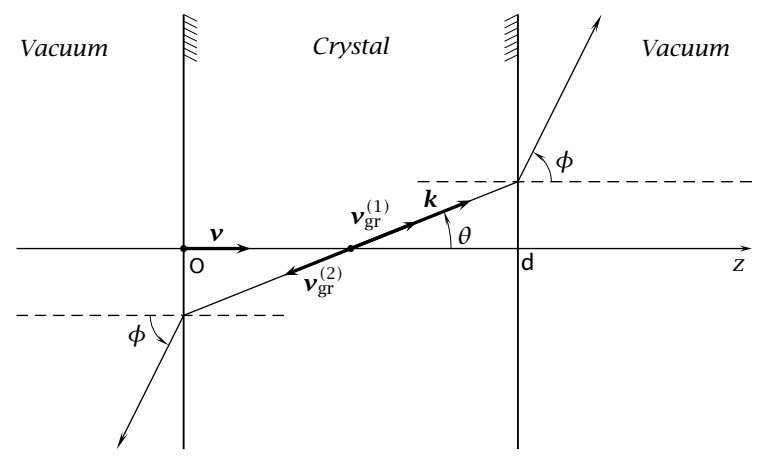

Figure 11. The direction of propagation of the waves of Cherenkov radiation, when spatial dispersion is taken into account, for the ordinary wave (subscript 1) and the anomalous wave (subscript 2) (Adapted from Ref. [53]).

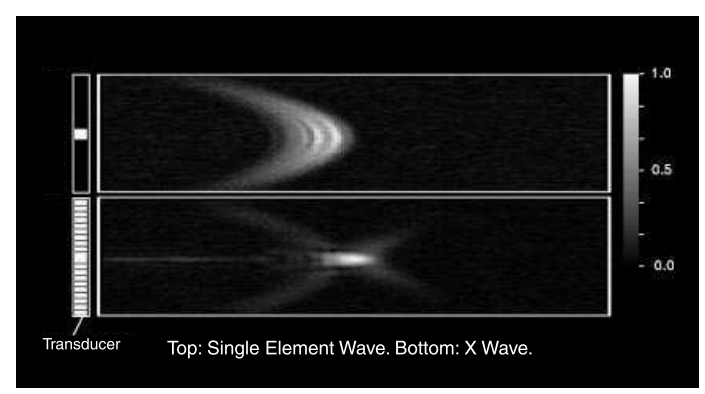

Figure 12. Normal sound propagation (upper figure), and the excitation interpreted as a supersonic soliton (lower figure); from reference [3] (We thank W. A. Rodrigues and J. E. Maiorino for providing us with a postscript file of this picture).

anharmonic interactions between the system and the surroundings) it is expected that complex behavior shall arise.

Resorting to an appropriate thermo-mechanical statistical approach, we have shown that such complex behavior consists of four particular phenomena. One is that the normal vibrational modes are accompanied by another type of excitation, consisting in the propagation of solitary waves of the Schrödinger-Davydov type. They are undeformed waves composed by a coherent state of normal modes. Although the wavepacket is spatially undeformed, it presents, as it should, decay in time with a given lifetime resulting from the dissipative effects that develop in the excited sample. The 
amplitude, velocity, and frequency of the solitary wave are determined by the initial and boundary conditions. Another phenomenon, arising out of the same nonlinearities that allows for the creation of the soliton, consists in that, under conditions of excitation which lead the system sufficiently away from equilibrium, there follows a large increase of the population of the modes lowest in frequency. This effect has a reminiscence of a Bose-Einstein condensation but here in nonequilibrium conditions, and we have termed it Fröhlich effect.

A third phenomenon consists in that, and again because of the nonlinearities which are responsible for both, Fröhlich effect and formation of a Schrödinger-Davydov soliton, the latter acquires a very long lifetime, that is, the soliton becomes nearly undamped, when travelling in the FröhlichBose-Einstein-like condensate.

Finally, the fourth phenomenon refers to a situation when, because of appropriate initial and boundary conditions, the soliton travels with a speed larger than that of the normal vibronic modes. In this case, as shown, there follows what can be termed as Fröhlich-Cherenkov effect: along two symmetrical privileged directions centered on the position of the soliton, is produced a large number of long wavelengths phonons. This could be the origin of the so-called X-waves observed in experiments of ultrasonography, as noticed in previous sections.
We conclude with the remark that solitary waves appear to be ubiquitous, and having large relevance in a number of important situations. Some at the technological level, like propagation in optical fibers (e.g., in a projected transAtlantic cable), in conducting polymers (for electric-car batteries; microcircuits; etc.), and the case of biological systems (long range propagation of nervous signals; the here mentioned case of medical imaging; etc.).

\section{Acknowledgments}

We gratefully acknowledge financial support to our Group, provided in different opportunities by the São Paulo State Research Foundation (FAPESP), the National Research Council (CNPq), the Ministry of Planning (Finep), Unicamp Foundation (FAEP), IBM Brasil, the John Simon Guggenheim Memorial Foundation (New York, USA).

\section{Appendix A The Acoustic Solitary Wave}

In direct space, after the terms that couple the amplitude $\left\langle a_{\vec{q}}\right\rangle$ with its conjugate are neglected, what, as noticed in the main text is accomplished using the rotating wave approximation, Eq. (14) takes the form:

$$
\begin{gathered}
\mathrm{i} \hbar \frac{\partial}{\partial t} \psi(x, t)=-\mathrm{i} \sum_{q} \hbar \omega_{\vec{q}} \int \frac{d x^{\prime}}{L} e^{\mathrm{i} q\left(x-x^{\prime}\right)} \psi\left(x^{\prime}, t\right)-\mathrm{i} \hbar \sum_{\vec{q}} \Gamma_{\vec{q}} \int \frac{d x^{\prime}}{L} e^{\mathrm{i} q\left(x-x^{\prime}\right)} \psi\left(x^{\prime}, t\right) \\
+\sum_{q_{1} q_{2}} R_{\vec{q}_{1} \vec{q}_{2}} \int \frac{d x^{\prime}}{L} \int \frac{d x^{\prime \prime}}{L} e^{i q_{1}\left(x-x^{\prime}\right)} e^{i q_{2}\left(x-x^{\prime \prime}\right)} \psi\left(x^{\prime}, t\right) \psi\left(x^{\prime \prime}, t\right) \psi^{*}(x, t),
\end{gathered}
$$

where, we recall, $\omega_{\vec{q}}=s q$. Considering that it is expected the formation of a highly localized packet (the soliton), centered in point $x$ and with a Gaussian-like profile with a width, say, $w$ (fixed by the initial condition of excitation) extending along a certain large number of lattice parameter $a$ (i.e. $w \gg a$ ), in Eq. (A.1) we make the expansion

$$
\psi\left(x^{\prime}, t\right) \approx \psi(x, t)-\xi \frac{\partial}{\partial x} \psi(x, t)
$$

where $\xi=x-x^{\prime}$ is roughly restricted to be smaller or at most of the order of $w$. The first term on the right of Eq. (A.1) is

$$
\begin{gathered}
\mathrm{i} \sum_{q} s|q| \int \frac{d x^{\prime}}{L} e^{\mathrm{i} q\left(x-x^{\prime}\right)} \psi\left(x^{\prime}, t\right) \\
=-\frac{L s}{2 \pi} \frac{\partial}{\partial x} \int_{0}^{\frac{\pi}{a}} d q \int_{0}^{L} \frac{d x^{\prime}}{L}\left[e^{\mathrm{i} q\left(x-x^{\prime}\right)}-e^{\mathrm{i} q\left(x-x^{\prime}\right)}\right] \psi\left(x^{\prime}, t\right) \\
\approx-\frac{\mathrm{i} s}{\pi} \frac{\partial}{\partial x} \int_{0}^{\frac{\pi}{a}} d q \int_{x-w / 2}^{x+w / 2} d \xi \sin (q \xi)\left[\psi(x, t)-\xi \frac{\partial}{\partial x} \psi(x, t)\right] \\
=\frac{\mathrm{i} s}{\pi} \frac{\partial}{\partial x}\left[\int_{x-\frac{1}{2} w}^{x+\frac{1}{2} w}\left(1-\cos \frac{\pi}{a} \xi\right) \frac{d \xi}{\xi}\right] \psi(x, t)
\end{gathered}
$$




$$
\begin{array}{r}
+\frac{\mathrm{i} s}{\pi}\left[\int_{x-\frac{1}{2} w}^{x+\frac{1}{2} w}\left(1-\cos \frac{\pi}{a} \xi\right) \frac{d \xi}{\xi}\right] \frac{\partial}{\partial x} \psi(x, t) \\
-\frac{\mathrm{i} s}{\pi}\left[\int_{x-\frac{1}{2} w}^{x+\frac{1}{2} w}\left(1-\cos \frac{\pi}{a} \xi\right) d \xi\right] \frac{\partial}{\partial x} \psi(x, t) \\
-\frac{\mathrm{i} s}{\pi}\left[\int_{x-\frac{1}{2} w}^{x+\frac{1}{2} w}\left(1-\cos \frac{\pi}{a} \xi\right) d \xi\right] \frac{\partial^{2}}{\partial x^{2}} \psi(x, t) .
\end{array}
$$

But, of the four terms after the last equal sign in this Eq. (A.3), the second and third are null, because of the Ansatz that a soliton would follow, since the derivative at the center of the packet is null. Consider now the last term, which after the integrations are performed becomes
But, we notice that the width of the packet is $w \gg a$, and the cosine in Eq. (A.4) has a period $2 a$, and then it oscillates very many times in $w$, and with amplitude $(2 a / \pi w) \ll 1$, and can be neglected. Similarly, the first term becomes proportional to

$$
-\frac{\mathrm{i} s w}{\pi}\left[1-\frac{2 a}{\pi w} \sin \frac{\pi}{a} \frac{w}{2} \cos \frac{\pi}{a} x\right] \frac{\partial^{2}}{\partial x^{2}} \psi(x, t) .
$$

$$
\text { is } \frac{\psi(x)}{\pi} \frac{x^{2}-(w / 2)^{2}}{x^{2}}\left\{w\left[1-\cos \left(\frac{\pi x}{a}\right) \cos \left(\frac{\pi w}{2 a}\right)\right]-2 x \sin \left(\frac{\pi x}{a}\right) \sin \left(\frac{\pi w}{2 a}\right)\right\},
$$

where, on the one hand, the oscillatory terms cancel on average, and, on the other hand, the term decays as $x^{-2}$. Consequently, using these results in Eq. (A.1), after introducing the notation $(\hbar s w / \pi) \equiv \hbar^{2} /\left(2 M_{s}\right)$, and the local approximation in the second and third term on the right of Eq. (A.1), we find Eq. 23.

\section{References}

[1] J. Lu and J. F. Greenleaf, Nondiffracting X-waves, IEEE Trans. Ultrason. Ferroelect. Freq. Contr. 39, 19 (1992).

[2] J. Lu and J. F. Greeleaf, Experimental Verification of Nondiffracting X-waves, IEEE Trans. Ultrason. Ferroelect. Freq. Contr. 39, 441 (1992).

[3] W. A. Rodrigues and J. Lu, On the Existence of Undistorted Progressive Waves, Found. Phys. 27, 435 (1997).

[4] A. Scott, F. Chu, and S. McLaughlin, The Soliton: A New Concept in Applied Science, Proc. IEEE 61, 1443 (1973).

[5] J. Scott-Russel, Report on Waves, Proc. Roy. Soc. Edinburgh, 319 (1844).

[6] A. C. Scott, The Solitary Wave, The Sciences (NYAS) 30(2), 28 (1990).

[7] T. Beardsley, Plastic Power, Scientific American 276(4), 39 (1997).

[8] A. J. Heger, S. Kivelson, J. R. Schrieffer, and W. P. Su, Solitons in Conducting Polymers, Rev. Mod. Phys. 60, 781 (1988)
[9] T. Anderson and S. Roth, Conducting Polymers: Electrical Transport and Current Applications, Braz. J. Phys. 24(3), 746 (1994).

[10] H. A. Haus, Molding light into solitons, IEEE Spectrum, 48 (March 1993).

[11] A. S. Davydov, Biology and Quantum Mechanics, Pergamon, Oxford, 1982.

[12] A. S. Davydov, Solitons in Biology, in Solitons, edited by S. E. Trullinger, V. E. Zakharov, and V. L. Prokovsky, chapter 1, pages 1-51, Elsevier, New York, 1986.

[13] A. C. Scott, Davydov's Soliton, Phys. Rep 217, 1 (1992).

[14] L. S. Garcia-Colin, A. R. Vasconcellos, and R. Luzzi, On Informational Statistical Thermodynamics, J. Non-Equilib. Thermodyn. 19, 24 (1994).

[15] A. R. Vasconcellos, R. Luzzi, and L. S. Garcia-Colin, A Microscopic Approach to IrreversibleThermodynamics (I): General Theory, Phys. Rev. A 43, 6622 (1991).

[16] A. R. Vasconcellos, R. Luzzi, and L. S. Garcia-Colin, A Microscopic Approach to Irreversible Thermodynamics (II): An Example from Semiconductor Physics, Phys. Rev. A 43, 6633 (1991).

[17] M. A. Tenan, A. R. Vasconcellos, and R. Luzzi, Mechanostatistical Foundations for Generalized Nonequilibrium Thermodynamics, Fortschr. Phys./Prog. Phys. 47, 1 (1996).

[18] R. Luzzi, A. R. Vasconcellos, D. Jou, and J. Casas-Vázquez, Thermodynamic Variables in the Context of a Nonequilibrium Ensemble Formalism, J. Chem. Phys. 107, 7383 (1997). 
[19] R. Luzzi, A. R. Vasconcellos, and J. G. Ramos, Statistical Foundations of Irreversible Thermodynamics, Teubner Bertelsmann-Springer, Berlin, 2000.

[20] R. Luzzi, A. R. Vasconcellos, and J. G. Ramos, Predictive Statistical Mechanics: A Nonequilibrium Ensemble Formalism, Kluwer Academic, Dordrecht, 2002.

[21] D. N. Zubarev, Nonequilibrium Statistical Thermodynamics, Consultants Bureau, New York, 1974, [Neravnovesnaia Statisticheskaia Termodinamika (Izd. Nauka, Moscow, 1971)].

[22] R. Luzzi and A. R. Vasconcellos, On the Nonequilibrium Statistical Operator Method, Fortschr. Phys./Prog. Phys. 38, 887 (1990).

[23] J. G. Ramos, A. R. Vasconcellos, and R. Luzzi, A Classical Approach in Predictive Statistical Mechanics: A Generalized Boltzmann Formalism, Fortschr. Phys./Prog. Phys. 43, 265 (1995).

[24] L. Lauck, A. R. Vasconcellos, and R. Luzzi, A Nonlinear Quantum Transport Theory, Physica A 168, 789 (1990).

[25] A. I. Akhiezer and S. V. Peletminskii, Methods of Statistical Physics, Pergamon, Oxford, 1981.

[26] A. C. Algarte, A. R. Vasconcellos, and R. Luzzi, Kinetic of Hot Elementary Excitations in Photoexcited Polar Semiconductors, Phys. Stat. Sol. (b) 173, 487 (1992).

[27] M. V. Mesquita, A. R. Vasconcellos, and R. Luzzi, Selective Amplification of Coherent Polar Vibrations in Biopolymers, Phys. Rev. E 48, 4049 (1993).

[28] M. V. Mesquita, A. R. Vasconcellos, and R. Luzzi, Neardissipationless Coherent Excitations in Biosystems, Int. J. Quantum Chem 60, 689 (1996).

[29] R. Luzzi, M. A. Scarparo, J. G. Ramos, A. R. Vasconcellos, M. L. Barros, Z. Zhiayo, and A. Kiel, Informational Statistical Thermodynamics and Thermal Laser Stereolithography, J. Non-Equilib. Thermodyn. 22, 197 (1997).

[30] A. R. Vasconcellos, M. V. Mesquita, and R. Luzzi, Solitary Wave Excitation in Acetanilide, Phys. Rev. Lett. 80, 2008 (1998).

[31] S. A. Hassan, A. R. Vasconcellos, and R. Luzzi, The informational-statistical-entropy operator in a nonequilibrium ensemble formalism, Physica A 262, 359 (1999).

[32] V. P. Kalashnikov, Equations of Motion, Green's Functions, and Thermodynamic Relations in Theories of Linear Relaxation, Theor. Math. Phys. 35, 362 (1978).

[33] K. Walyazek, D. N. Zubarev, and A. Z. Kuzemskii, Schrödinger-type equation with damping for a dynamical system in a thermal bath, Theor. Math. Phys. 5, 1150 (1971).

[34] H. Fröhlich, Long Range Coherence and the Action of Enzymes, Nature 228, 1093 (1970).

[35] H. Fröhlich, The Biological Effects of Microwaves and Related Questions, in Advances in Electronics and Electron Physics, volume 17, pages 85-152, Academic Press, New York, 1980

[36] H. Haken, Handbuch der Physik XXV/2C, Springer, Berlin, 1970.

[37] V. E. Zakharov, The Inverse Scattering Method, in Solitons, edited by R. K. Bullough and P. J. Caudrey, chapter 7, pages 243-285, Springer, Berlin, 1980.
[38] G. Careri, U. Buotempo, R. Carta, E. Gratton, and A. C. Scott, Infrared Absorption in Acetanilide by Solitons, Phys. Rev. Lett. 51(4), 304 (1983).

[39] J. L. Sauvajol, R. Almaraic, J. Moret, M. Barthes, and J. L. Ribet, Temperature Dependence of the Raman spectrum of fully deuterated acetanilide, J. Raman Spectrosc. 20, 517 (1989).

[40] G. Careri and E. Gratton, Fine Structure of the Amide I Band in Acetanilide, Phys. Rev. A 37(10), 4048 (1988).

[41] M. Sakai, N. Kuroda, and Y. Nishima, High-pressure Raman Study of Vibrational Spectra in Crystalline Acetanilide, Phys. Rev. B 47(1), 150 (1993).

[42] M. Barthes, R. Almaraic, J. L. Sauvajol, , R. Currat, J. Moret, and J. L. Ribet, Neutron scattering investigation of deuterated crystalline acetanilide, Europhys. Lett. 7, 55 (1988).

[43] M. Barthes, J. Eckert, S. W. Johnson, J. Moret, B. I. Swanson, and C. J. Unjeker, Anomalous Vibrational Modes in acetanilide as studied by inelastic neutron scattering, J. Phys. I France 2, 1929 (1992).

[44] R. Luzzi and A. R. Vasconcellos, Response Function Theory for Far-from-Equilibrium Systems, J. Stat. Phys. 23, 539 (1980).

[45] V. P. Kalashnikov, Response to a Mechanical Perturbation and the Green's Functions for Nonequilibrium Systems, Soviet Phys.-Usp. 9, 94 (1971).

[46] M. V. Mesquita, A. R. Vasconcellos, and R. Luzzi, Solitons in highly excited matter: Dissipative thermodynamics and supersonic effects, Phys. Rev. E 58(6), 7913 (1998).

[47] I. Prigogine, Structure, Dissipation, and Life, in From Theoretical Physics to Biology, edited by M. Marois, North Holland, Amsterdam, 1969.

[48] G. Nicolis and I. Prigogine, Self-organization in Nonequilibrium Systems, Wiley-Interscience, New York, 1977.

[49] R. Luzzi and A. R. Vasconcellos, Dissipation and Order in Complex Systems: An overview, Ciência e Cultura 43, 423 (1992), A publication of the Brazilian Society for the Advancement of Science.

[50] J. M. Ziman, Electrons and Phonons, Clarendon, Oxford, 1960.

[51] L. D. Landau and E. M. Lifshitz, Electrodynamics of Continuous Media, Pergamon, Oxford, 1960.

[52] V. L. Ginsburg, Certain Theoretical Aspects of Radiation due to Superluminal Motion in a Medium, Physics-Uspekhi 2, 874 (1960).

[53] V. M. Agranovich and V. L. Ginsburg, Spatial Dispersion in Crystal Optics and the Theory of Excitons, WileyInterscience, London, 1966.

[54] P. Kocevar, Hot phonon dynamics, Physica B 34, 155 (1985).

[55] P. T. Landsberg, Photons at non-zero chemical potential, J. Phys. C: Solid State Phys. 14, L1025 (1981).

[56] J. Lu, Z. Hehong, and J. F. Greenleaf, Biomedical ultrasound beam forming, Ultrasound Med. Biol. 20, 403 (1994). 